\title{
CpG Methylation of the GPX3 Promoter in Patients with Kashin-Beck Disease Potentially Promotes Chondrocyte Apoptosis
}

\section{Rongqiang Zhang}

Shaanxi University of Chinese Medicine

\section{Di Zhang}

Xi'an Jiaotong University

\section{Xiaoli Yang}

Xi'an Jiaotong University

\section{Dandan Zhang}

Xi'an Jiaotong University

\section{Qiang Li}

Xi'an Jiaotong University

\section{Chen Wang}

Xi'an Jiaotong University

\section{Xuena Yang}

Xi'an Jiaotong University

Yongmin Xiong ( $\nabla$ xiongym@mail.xjtu.edu.cn )

Xi'an Jiaotong University https://orcid.org/0000-0003-3351-2128

\section{Research article}

Keywords: Kashin-Beck Disease, GPX3, DNA methylation, CpG

Posted Date: January 13th, 2021

DOI: https://doi.org/10.21203/rs.3.rs-142170/v1

License: (c) (1) This work is licensed under a Creative Commons Attribution 4.0 International License.

Read Full License

Version of Record: A version of this preprint was published at Journal of Trace Elements in Medicine and Biology on February 1st, 2022. See the published version at https://doi.org/10.1016/j.jtemb.2022.126943. 


\section{Abstract}

Objective: To determine the methylation levels of $\mathrm{CpGs}$ in the GPX3 promoter region and explore their potential effects on the apoptosis of chondrocytes.

Methods: Blood specimens were collected from 32 participants, including $16 \mathrm{KBD}$ patients and 16 healthy subjects. Twenty-five CpGs in the promoter region of GPX3 were identified and detected by MALDI-TOFMS. Methylation levels of $\mathrm{CpGs}$ were compared between KBD patients and healthy subjects as well as among the KBD patients with different degrees. C28/I2 human chondrocytes were treated with tBHP and $\mathrm{Na}_{2} \mathrm{SeO}_{3}$. Apoptosis in chondrocytes was examined under a fluorescence microscope.

Results: The methylation levels of GPX3-8_CpG_11, GPX3-8_CpG_16, GPX3-8_CpG_20 in KBD patients were significantly higher than those of healthy subjects $(P<0.05)$. The methylation levels of the other CpGs were not significantly different between the two groups ( $P>0.05)$. The methylation level of GPX38_CpG_24 in KBD patients with degree III was significantly higher than those in KBD patients with degree $\mathrm{I} / \mathrm{II}(P<0.05) \mathrm{MSP}-\mathrm{PCR}$ analysis indicated that the methylation rate of KBD group $(9.41 \%)$ was significantly higher than that of healthy subjects (1.18\%), and that GPX3 DNA methylation increased the risk of acquiring $\mathrm{KBD} 8$ fold $(\mathrm{OR}=8.000,95 \% \mathrm{Cl}: 1.023-62.580)$; The mRNA expression of GPX3 in whole blood of KBD patients was lower than that of healthy subjects ( $P \otimes 0.05)$; Compared with the control group, $G P X 3, G P X 1$ and GPX4 mRNA level of the tertbutyl hydroperoxide injury group decreased significantly ( $P$ $<0.05)$, after supplementation with $\mathrm{Na}_{2} \mathrm{SeO}_{3}$. The logarithmic increase in mRNA levels of GPX3, GPX1 and GPX4 decreased the rate of apoptosis in chondrocytes.

Conclusion: The methylation patterns of CpGs in GPX3 varied in KBD patients. The experiments indicated that the increased methylation of $\mathrm{CpGs}$ within the GPX3 promoter may down-regulate the expression of $G P X 3$, thereby reducing the antioxidant function of GPX3 and promoting chondrocyte apoptosis, both of which accelerates the development of KBD.

\section{Background}

Kashin-Beck disease (KBD) is a common endemic, chronic and degenerative bone disease with the highest prevalence in China. KBD mainly affects the joints and cartilages of individuals[1,2].

Geographically, KBD is mainly distributed from the northeast to the southwest of China in a belt shaped manner, as well as parts of Eastern Siberia in Russia, and North Korea[3, 4]. The recent epidemiological survey indicated that 0.54 million individuals were suffering from KBD by the close of 2017. Currently, it is estimated that 37.2 million individuals are at high risk of developing KBD in China[5]. KBD gravely threatens the health of the population; however, its pathogenesis remains elusive.

Seleniun deficiency has been proposed for decades as the archetypal etiological theory of KBD[6-8]. However, the roles of selenium in the occurrence and development of KBD has not been ascertained. Selenium usually performs its biological roles in the human body as selenoprotein, of which its physiological function still needs to be systematically studied. The antioxidant effect of selenoprotein is 
mainly attributed to the oxidation state of the selenium atom. Such antioxidant enzymes prevents DNA damage caused by oxidative stress, which is related to upregulated apoptosis and necrosis of KBD articular chondrocytes[9-11]. The above evidence indicates that the antioxidant properties of selenoproteins can protect chondrocytes from apoptosis and necrosis.

In recent years, studies have confirmed that, despite low environmental selenium concentrations, the individual's genetic characteristics also play an important role in the occurrence and development of $\mathrm{KBD}$ [12]. Therefore, the genetic factors contributing to KBD have received increasing attention. Consequently, a number of KBD susceptible genes were identified from some studies through modern molecular biology research technologies[13-15]. However, studies on the genetic factors contributing to KBD can only explain its pathogenesis partly. Researchers are rapidly discovering that epigenetic elements may also affect gene function by regulating gene expression levels[16], hence exhibiting a series of effects on human physiology.

Glutathione peroxidase family are key members of selenoproteins that catalyze the reduction of organic hydroperoxides and hydrogen peroxide $\left(\mathrm{H}_{2} \mathrm{O}_{2}\right)$ by glutathione, and thereby inhibiting oxidative cell damage[17, 18]. Experiments have showed that downregulation of GPX3 expression by hypermethylation of its promoter, has been observed in a wide spectrum of human malignancies such as Osteoarthritis $(\mathrm{OA})$ and KBD[16]. Therefore, genetic and epigenetic interactions of GPX3 may affect human health. However, methylation levels of the GPX3 promoter has not been quantified in KBD.

In our present study, CpGs of GPX3 in the promoter region were identified and determined. Matrix assisted laser desorption/ionization time of flight mass spectrometry (MALDI-TOF-MS) was employed to quantitate the methylation of $\mathrm{CpGs}$ explored their potential effect on the apoptosis of chondrocytes. Through these evaluations, the current study aimed to identify the divergent methylation of CpGs in GPX3 promoter regions in KBD patients, and to further analyze the effect of GPX3 promoter methylation in KBD.

\section{Methods And Materials}

\subsection{Study population}

The study population was selected from KBD endemic areas of Shaanxi province, China. A biological specimen library was created from blood samples drawn from 226 KBD patients and 248 healthy subjects (Table 1). All the study participants received prudent radiographic examination. The national diagnosis criteria of KBD (WS/T207-2010) was used to select patients with KBD. Individuals with genetic bone and cartilage diseases, arthritis related disease and other skeletal disorders were excluded from this study. Individuals who met the selection criteria were recruited to participate in the study after submitting their signed informed consent. The proposed study design met the approval of the Human Ethics Committee of Xi'an Jiaotong University, People's Republic of China.

\subsection{Quantifying the mRNA expression in KBD patients and chondrocytes}


Total RNA from KBD patients and controls ( $n=8$ in each group), as well as chondrocytes ( $n=3$ in each group), was extracted using the Trizol KIT (Life Technologies, Carlsbad, CA). RNA extracts were reversetranscribed into cDNA using the RevertAidTM First Strand cDNA Synthesis Kit (MBI, Fermentas, Vilnius, Lithuania) following the manufacturer's instructions. Relative quantification of GPX3, GPX1 and GPX4 mRNA was performed by iQe5 quantitative real-time PCR Detection Systems (qRT-PCR) (Bio-Rad, Philadelphia, PA) with $\beta$-actin as a reference. The sequences of primers are listed in Table 2. qRT-PCR was performed in a $20 \mu \mathrm{L}$ reaction mixture containing cDNA $(1.6 \mu \mathrm{L})$, each primer $(0.8 \mu \mathrm{L}), 2 \times$ SYBR Premix Ex Taq $\otimes(10 \mu \mathrm{L})$ (Takara, Mountain View, CA), and $\mathrm{ddH}_{2} \mathrm{O}(6.8 \mu \mathrm{L})$ using the TaqMan method $\left(94^{\circ} \mathrm{C}\right.$ for 2 min, and 40 cycles of $94^{\circ} \mathrm{C}$ for $10 \mathrm{~s}$ and $72^{\circ} \mathrm{C}$ for $30 \mathrm{~s}$ ). All reactions were performed in duplicate. Relative expression levels of GPX3, GPX1 and GPX4 mRNAs were normalized to $\beta$-actin and analyzed by iQe5 software (version 2.0, Bio-Rad, Philadelphia, PA).

\subsection{Quantitative methylation analysis}

\subsubsection{The design and synthesis of primers}

Primers covering $\mathrm{CpGs}$ for quantitative methylation analysis were designed by Agena's software (http://www.epidesigner.com/index.html) and synthesized by Liuhe Huada Gene Technology Co., Ltd (Beijing, China). The primer sequences are as follows: the forward primer, Fw: GGAATAAGAAATGTTTTTTAGAATGGA, and the reverse primer, Rv: ACCAAAAACAAAAAAAACAAACAAA, which are also illustrated in Figure 1A. The target fragment of GPX3 contained $25 \mathrm{CpGs}$, which is showed in Figure 1B.

\subsubsection{Quantitative methylation by MALDI-TOF MS}

EZ-96 DNA methylation kit (Zymo Research) was used to treat the genomic DNA (200 ng) of each participant with bisulfite according to the manufacturer's instructions. The Sequenom Mass ARRAY platform (CapitalBio, Beijing, China) containing a matrix-assisted laser desorption/ionization time-offlight (MALDI-TOF) mass spectrometer and RNA base-specific cleavage (Mass CLEAVE), were both employed to quantify the methylation of GPX3 CpGs. Quantitative methylation data was obtained via a Spectro CHIP (SEQUENOM) and a Mass ARRAY Compact System (SEQUENOM). The EpiTYPER software version 1.0 (SEQUENOM) was used to analyze and display the results.

\subsection{Qualitative methylation analysis}

Genomic DNA of the study populationwas extracted from whole blood samples ( $n=80$ in each group) using TIANamp Genomic DNA Kit (Tiangen Biotech, Beijing, China). The genomic DNA was treated with bisulfite using EZ-96 DNA methylation kit (ZYMO Research, Irvine, CA) according to the manufacturer's instructions and amplified by methylation-specific polymerase chain reaction (MSP). The sequences of primer are as follows: methylated primers (F: 5'-TATGTTATTGTCGTTTCGGGAC-3'; R: 5'GTCCGTCTAAAATATCCGACG-3', products size: 177bp) and unmethylated primers (F: 5'-

TTTATGTTATTGTTGTTTTGGGATG-3'; R: 5'-ATCCATCTAAAATATCCAACACTCC-3', products size: 186bp). 
Next, a $50 \mu \mathrm{L}$ PCR mixture containing $5 \mu \mathrm{L} 10 \times P C R$ buffer, $4 \mu \mathrm{L}$ dNTP mixture, $1 \mu \mathrm{L}$ of each primer, $3 \mu \mathrm{L}$ bisulfite-modified DNA, 35.75 $\mathrm{L}$ ddH2O, and $0.25 \mu \mathrm{L}$ hot-start Taq-polymerase (Takara, Mountain View, CA) for each blood sample was prepared. PCR conditions were set as: $94^{\circ} \mathrm{C}$ for 10 min (initial denaturation), followed by 40 cycles of $94^{\circ} \mathrm{C}$ for $30 \mathrm{~s}, 60^{\circ} \mathrm{C}$ for $30 \mathrm{~s}$ and $72{ }^{\circ} \mathrm{C}$ for $30 \mathrm{~s}$, and a final extension of $72{ }^{\circ} \mathrm{C}$ for 10 min. Finally, PCR products were resolved in $2 \%$ agarose gels, stained with ethidium bromide, and visualized in ultraviolet light (GBox F3, Syngene, UK).

\subsection{C28/I2 human chondrocytes injured by tBHP}

Human chondrocyte cell line named C28/I2, was provided by Professor Mary B Goldring (Hospital for Special Surgery, Weill Cornell Medical College, New York, USA). C28/I2 was cultured in DMEM/F-12 (Hyclone, Logan, UT) supplemented with 12\% FBS (SiJiQing, Zhejiang, China) and 1\% penicillin/streptomycin solution in a humidified incubator at $37^{\circ} \mathrm{C}$ and $5 \% \mathrm{CO}_{2}$. Upon reaching $90 \%$ confluence, chondrocytes were seeded on 96-well culture plates. Our experiment contained 6 groups: control group (C), purely Selenium (Se) group ( $2,0.10 \mu \mathrm{g} / \mathrm{mL} \mathrm{Na}_{2} \mathrm{SeO}_{3}$ ), tertbutyl hydroperoxide (tBHP) injury group (0, $150 \mu \mathrm{mol} / \mathrm{L}$ tBHP), low Se group (OS1, $\left.0.05 \mu \mathrm{g} / \mathrm{mL} \mathrm{Na}_{2} \mathrm{SeO}_{3}+150 \mu \mathrm{mol} / \mathrm{L} \mathrm{tBHP}\right)$, medium Se group (OS2, $\left.0.10 \mu \mathrm{g} / \mathrm{mL} \mathrm{Na}_{2} \mathrm{SeO}_{3}+150 \mu \mathrm{mol} / \mathrm{L} \mathrm{tBHP}\right)$ and high Se group (OS3, $0.15 \mu \mathrm{g} / \mathrm{mL}$ $\left.\mathrm{Na}_{2} \mathrm{SeO}_{3}+150 \mu \mathrm{mol} / \mathrm{L} \mathrm{tBHP}\right)$. OS1, OS2 and OS3 were treated with increasing concentrations of $\mathrm{Na}_{2} \mathrm{SeO}_{3}(0.05,0.10$ and $0.15 \mu \mathrm{g} / \mathrm{mL})$ for $24 \mathrm{~h}$ as pre-protection, and then treated with $150 \mu \mathrm{mol} / \mathrm{L} \mathrm{tBHP}$ for $24 \mathrm{~h}$. Chondrocytes were stained with $2 \%$ Hoechst 33342 in DMEM/F-12 containing $12 \%$ FBS and incubated at $37^{\circ} \mathrm{C}$ for $30 \mathrm{~min}$. Apoptosis in chondrocytes was monitored under a fluorescence microscope. Five high-power fields (100x) were selected randomly to count apoptotic cells and calculate the apoptotic rate in each group $(n=3)$.

\subsection{Statistical analysis}

Quantitative data was presented as mean \pm standard deviation (SD). Groups were compared using the student's $t$-test. Curve-fitting method was used for obtaining the correlation between GPX1, GPX3 and GPX4 mRNA expressions and apoptosis rate of chondrocytes. All statistical analyses were performed using SPSS 23.0, and the significance level $\mathrm{a}=0.05$ were considered to have statistical significance (SPSS Inc., Chicago, IL, USA).

\section{Results}

\subsection{Baseline characteristics of study population}

$226 \mathrm{KBD}$ patients and 248 healthy controls with matched frequency of age and gender were recruited in this study. No statistically significant differences were seen between the cases and controls in age $(t=1.305, P=0.193)$ and $\operatorname{sex}\left(x^{2}=1.914, P=0.167\right)$. The demography of the two groups are shown in Table 1 . 
$16 \mathrm{KBD}$ patients and 16 healthy subjects with no significant differences in age and gender, were selected randomly from the study population to detect $\mathrm{CpG}$ methylation detection (Table 3). Figure 2 illustrates the methylation profiles of $14 \mathrm{CpGs}$ (or CpG Units) in the promoter region of GPX3. The methylation levels of GPX3-8_CpG_11, GPX3-8_CpG_16, GPX3-8_CpG_20 in KBD patients were significantly higher than those in the healthy subjects $(P<0.05)$. The methylation levels of the other $\mathrm{CpGs}$ were not significantly different between the two groups $(P>0.05)$ (Figure 3$)$.

\subsection{The CpGs methylation of GPX3 in KBD patients with different degrees}

The methylation levels of $14 \mathrm{CpGs}$ (or CpG Units) in the promoter region of GPX3 between KBD patients with degree I/II and III are shown in Figure 4. The methylation level of GPX3-8_CpG_24 in KBD patients with degree III was significantly higher than those in KBD patients with degree I/II $(P<0.05)$.

\subsection{Validation by MSP-PCR}

MSP-PCR was used to detect the methylation status of GPX3promotor region in KBD patients and healthy subjects ( $n=85 /$ group) quantitatively (Table 4 ). The amplicons were resolved by $2 \%$ agarose gel electrophoresis with band patterns showing that 8 (9.41\%) patients with KBD and $1(1.18 \%)$ healthy controls developed methylation in GPX3. After $x^{2}$ test, the difference of methylation rates of GPX3 between the two groups was significant $\left(x^{2}=4.396, P=0.036\right)$, indicating that the methylation rate of KBD group was significantly higher than that of control group. Also, GPX3 DNA methylation increased the risk of KBD 8-fold (OR $=8.000,95 \% \mathrm{Cl}$ : 1.023-62.580) (Table 5). The results of MSP-PCR and MALDI-TOFMS were harmonious.

\subsection{The mRNA transcription level of GPX3 in KBD patients}

GPX3 mRNA level in whole blood was detected by qRT-PCR with $\beta$-actin as a quantitative control. Results showed the mRNA expression of GPX3 in whole blood of KBD patients was lower than that of healthy subjects (Pख0.05) (Figure 5).

\subsection{The effect of Oxidative damage and Selenium on chondrocytes apoptosis}

The results showed that the apoptosis rate in group C, O, S2, OS1, OS2, OS3 was $(1.30 \pm 0.32) \%,(65.96 \pm$ $3.98) \%,(2.03 \pm 0.36) \%,(14.54 \pm 2.02) \%,(4.15 \pm 0.19) \%$, and $(14.74 \pm 2.56) \%$, respectively. The apoptosis rates of the six groups was significantly different from each other $(F=595.157, P<0.001)$. Pairwise comparison using LSD analysis suggested that the apoptosis rate in group 0 was significantly higher than that in group $C(P<0.001)$ while apoptosis rates of group OS1, OS2, OS3 were significantly lower than that of group $O(P<0.001)$. These results indicate that tBHP-induced oxidative damage can increase apoptosis of chondrocytes, while in contrast, supplementation with $\mathrm{Na}_{2} \mathrm{SeO}_{3}$ can decrease apoptosis of chondrocytes.

\subsection{The mRNA transcription levels of GPX3 in chondrocytes}


The changes of GPX3 mRNA levels in chondrocytes treated with tBHP and $\mathrm{Na}_{2} \mathrm{SeO}_{3}$ was further examined. The results showed that GPX3 mRNA level of group 0 decreased significantly compared with group $\mathrm{C}(P<0.05)$. However, GPX3 mRNA levels were elevated upon supplementation with $\mathrm{Na}_{2} \mathrm{SeO}_{3}$ (Figure 6A).

\subsection{The mRNA transcription levels of GPX1 and GPX4 in chondrocytes}

The changes in mRNA levels of GPX1 and GPX4, the two main members of GPXs family, in chondrocytes treated with tBHP and $\mathrm{Na}_{2} \mathrm{SeO}_{3}$ was also examined to observe whether they exhibited similar trends in mRNA transcription. The results showed that GPX1 and GPX4 mRNA levels both decreased significantly in group 0 in comparison with group $C(P<0.05)$. However, GPX1 and GPX4 mRNA levels were increased after supplementation with $\mathrm{Na}_{2} \mathrm{SeO}_{3}$ (Figure 6B-C).

\subsection{The effect of GPX1, GPX3 and GPX4 mRNA transcription levels on chondrocytes apoptosis}

In order to understand the effect of GPX1, GPX3 and GPX4 mRNA levels on chondrocytes apoptosis, curve fitting was used to correlate $G P X 1, G P X 3, G P X 4$ mRNA levels with apoptosis rate of chondrocytes. The results showed that the rate of apoptosis was decreased with the elevation of GPX1, GPX3, GPX4 mRNA levels. In addition, the increasing trends of GPX1, GPX3, GPX4 mRNA levels exhibited similar "L" curves (Figure 7).

\section{Discussion}

We aimed to evaluate the $\mathrm{CpG}$ methylation level of GPX3 of KBD quantitatively. Primers targeting the 25 $\mathrm{CpGs}$ were therefore designed to test the $\mathrm{CpG}$ methylation levels by MALDI-TOF-MS. Significant variation in methylation of GPX3 CpGs in KBD blood were identified. To substantiate the pathogenetic implication for KBD, C28/I2 human chondrocytes treated with tBHP were cultured to explore the potential effect of methylation patterns in the $\mathrm{CpGs}$ of GPX3 on KBD development. The results showed that the mRNA transcription levels of GPX3 in KBD patients and chondrocytes treated with tBHP were all decreased significantly, similarly, the apoptosis rates of chondrocytes were decreased with logarithmic elevation of GPX3 mRNA levels. The results indicated that increased levels of methylation in GPX3 CpGs may downregulate the GPX3 mRNA transcription levels, thereby reducing its antioxidant function, promoting apoptosis of chondrocytes and consequently accelerating the development of KBD.

Classic chondrocytic necrosis in articular cartilage, excessive apoptosis, dedifferentiation of chondrocytes, and chronic inflammation are the main pathological features of KBD, which are widely accepted. Findings about the beneficial effects of various antioxidants on KBD are gradually increasing. Selenium, assimilated in humans as selenocysteine (Sec) in selenoproteins such as glutathione peroxidases(GPXs), participates in antioxidant defense in human bodies[19]. More than 50 selenoproteins have currently been identified and 25 of them can be found in humans $[19,20]$. Nevertheless, many of their structure and specific biological functions remains an enigma. Glutathione 
peroxidases (GPXs) are a big family of antioxidant enzymes which can actively reduce $\mathrm{H}_{2} \mathrm{O}_{2}$ and organic hydroperoxides to inhibit the ROS-induced damage to cell membranes and proteins[21]. Possibly, GPXs protect cells from oxidative damage. In mammals, eight glutathione peroxidases (GPX1-GPX8) have been identified so far, of which GPX1-GPX4 and GPX6 are selenium-containing GPXs[22].

Currently and collectively, the biological functions of GPX1, GPX3, and GPX4 are widely and mostly studied. GPX1 is activated during cartilage formation and is responsible for eliminating reactive oxygen species (ROS), which are essential for chondrogenic induction[23]. Results reported here showed that GPX1 knockdown can impair gene expression of Col II and aggrecan and also suppress the proliferation of chondrocytes. Therefore, GPX1 is necessary for chondrogenic induction in ATDC5 cells[24]. Another study showed that GPX3 was significantly increased in bone samples from hip fracture patients, which suggested increased antioxidative activity in the samples[25]. The latest evidence showed that the methylation and expression of GPX3 vary in KBD blood samples[16], which may have an important role in this bone disease occurrence and development. Furthermore, the mRNA level of GPX4 was strikingly decreased in the blood of KBD patients and the polymorphisms of two SNPs (rs713041, rs4807542) of GPX4 may be related to the development of KBD. On that account, GPX4 can serve as a potential susceptibility biomarker for $\mathrm{KBD}[26]$. The above evidence strongly suggests that these seleniumcontaining GPXs may play essential roles in the occurrence of KBD, which is worth exploring further.

In addition to the genetic factors of GPXs, their epigenetic studies are also an important hotspot for study. However, quantitative research on the methylation levels of GPXs in KBD have not been reported. In our present study, we paid special attention to the quantitative assessment of GPX3 CpG methylation levels and their potential effect on apoptosis of chondrocytes. The results indicated that increased levels of GPX3 CpGs methylation may down-regulate GPX3 mRNA transcription and induce chondrocyte apoptosis. Our study provided valuable clues for exploring the potential interaction between the CpG methylation (epigenetics) and mRNA expression (genetics) of GPX3 in KBD. Integrated analysis of the genetic and epigenetics information of genes will unravel the complexity of chronic bone diseases, such as $\mathrm{KBD}[27,28]$.

It has been recognized that epigenetic deregulation through genetic and environmental elements could result in delayed or blocked cancer development[29]. The genetic profile of an individual could provide the underlying susceptibility factors and triggers for the evolution of a complex disease (cancer, bone disease, etc.). Epigenetic regulation also has very important implications for development of such diseases[30]. Increasing evidence showed that unraveling the interplay between genetic and epigenetic parameters becomes increasingly essential for interpreting the etiology of complex diseases[27, 28, 30]. The findings of our present study provide preliminary clues and new evidence that could illustrate the interplay between genetic and epigenetic parameters of GPX 3 in the onset of KBD.

\section{Conclusions}


The methylation profiles of $\mathrm{CpGs}$ in the promoter region of GPX3 in the whole blood of KBD patients were detected and their potential effect on the apoptosis of chondrocytes was explored. It was initially suggested that there may be an interplay between the CpGs methylation in the promoter region of GPX3 and GPX3 expression in KBD patients, which stimulated chondrocyte apoptosis. Our present study provides a new perspective and new clue for mastering the pathogenic process of KBD.

\section{Declarations}

\section{Ethics approval and consent to participate}

Individuals were recruited to participate in the study after submitting their signed informed consent. The proposed study design met the approval of the Human Ethics Committee of Xi'an Jiaotong University, People's Republic of China.

\section{Consent for publication}

Not applicable.

\section{Availability of data and material}

All data generated or analyzed during this study are included in this published article.

\section{Authors' contributions}

Study design and conception: Yongmin Xiong, Rongqiang Zhang

Acquisition of the data: Rongqiang Zhang, Di Zhang, Xiaoli Yang,Dandan Zhang, Qiang Li, Chen Wang, Xuena Yang

Analysis and interpretation of the data: Rongqiang Zhang, Yongmin Xiong

All authors were involved in reading the draft and revising it carefully, and all authors approved the final version to be published.

\section{Competing interests}

The authors declared no conflict of interest.

\section{Funding}

This study is supported by the National Natural Science Foundation of China (81773372, 81573104), Key Research and Development Program in Shaanxi Province (2020SF-076), Research Project from Health Commission of Shaanxi Provincial Government (2018A017), Education Department of Shaanxi Provincial Government (19JS015), Special R\&D Program Project of Chinese Academy of Se-enriched Industry (2020FXZX05-01). 


\section{Acknowledgements}

We are grateful to the participants, Chao Lu at the Honghui Hospital Affiliated to Xi'an Jiaotong University for providing the clinical material. The study was supported by National Natural Science Foundation of China (81773372, 81573104), Key Research and Development Program in Shaanxi Province (2020SF076), Research Project from Health Commission of Shaanxi Provincial Government (2018A017), Education Department of Shaanxi Provincial Government (19JS015).

\section{References}

1. Shi, Z., et al., Environmental water chemistry and possible correlation with Kaschin-Beck Disease (KBD) in northwestern Sichuan, China. Environ Int, 2017. 99: p. 282-292.

2. Guo, Y., et al., Trace Element Levels in Scalp Hair of School Children in Shigatse, Tibet, an Endemic Area for Kaschin-Beck Disease (KBD). Biol Trace Elem Res, 2017. 180(1): p. 15-22.

3. Song, Q.Q., et al., The urinary levels of CTX-II, C2C, PYD, and Helix-II increased among adults with KBD: a cross-sectional study. J Orthop Surg Res, 2019. 14(1): p. 328.

4. Fu, Q., et al., Radiographic features of hand osteoarthritis in adult Kashin-Beck Disease (KBD): the Yongshou KBD study. Osteoarthritis Cartilage, 2015. 23(6): p. 868-73.

5. NHFPC, China Health and Family Planning Statistical Yearbook. 2018, Beijing: Peking Union Medical College Press.

6. Sahebari, M., Z. Rezaieyazdi, and M. Khodashahi, Selenium and Autoimmune Diseases: A Review Article. Curr Rheumatol Rev, 2019. 15(2): p. 123-134.

7. Vinceti, M., T. Filippini, and L.A. Wise, Environmental Selenium and Human Health: an Update. Curr Environ Health Rep, 2018. 5(4): p. 464-485.

8. Fang, W., et al., Environmental Se-Mo-B deficiency and its possible effects on crops and Keshan-Beck disease (KBD) in the Chousang area, Yao County, Shaanxi Province, China. Environ Geochem Health, 2003. 25(2): p. 267-80.

9. Wang, W., et al., Oxidative stress and status of antioxidant enzymes in children with Kashin-Beck disease. Osteoarthritis Cartilage, 2013. 21(11): p. 1781-9.

10. Dai, X., et al., Effects of sodium selenite on c-Jun N-terminal kinase signalling pathway induced by oxidative stress in human chondrocytes and c-Jun N-terminal kinase expression in patients with Kashin-Beck disease, an endemic osteoarthritis. Br J Nutr, 2016. 115(9): p. 1547-55.

11. Zhang, R., et al., Pathway-based network analyses and candidate genes associated with Kashin-Beck disease. Medicine (Baltimore), 2019. 98(18): p. e15498.

12. Wen, Y., et al., Gene expression analysis suggests bone development-related genes GDF5 and DIO2 are involved in the development of Kashin-Beck disease in children rather than adults. PLoS One, 2014. 9(7): p. e103618. 
13. Zhang, F., et al., Trans-omics pathway analysis suggests that eQTLs contribute to chondrocyte apoptosis of Kashin-Beck disease through regulating apoptosis pathway expression. Gene, 2014. 553(2): p. 166-9.

14. Zhang, F., et al., Expression profile analysis of mycotoxin-related genes in cartilage with endemic osteochondropathy Kashin-Beck Disease. BMC Musculoskelet Disord, 2012. 13: p. 130.

15. Zhang, F., et al., Genome-wide gene expression analysis suggests an important role of hypoxia in the pathogenesis of endemic osteochondropathy Kashin-Beck disease. PLoS One, 2011. 6(7): p. e22983.

16. Han, L., et al., The study of GPX3 methylation in patients with Kashin-Beck Disease and its mechanism in chondrocyte apoptosis. Bone, 2018. 117: p. 15-22.

17. Drevet, J.R., The antioxidant glutathione peroxidase family and spermatozoa: a complex story. Mol Cell Endocrinol, 2006. 250(1-2): p. 70-9.

18. Zachara, B.A., et al., Red blood cell and plasma glutathione peroxidase activities and selenium concentration in patients with chronic kidney disease: a review. Acta Biochim Pol, 2006. 53(4): p. 663-77.

19. Lobanov, A.V., D.L. Hatfield, and V.N. Gladyshev, Eukaryotic selenoproteins and selenoproteomes. Biochim Biophys Acta, 2009. 1790(11): p. 1424-8.

20. Guo, L., et al., Selenocysteine-Specific Mass Spectrometry Reveals Tissue-Distinct Selenoproteomes and Candidate Selenoproteins. Cell Chem Biol, 2018. 25(11): p. 1380-1388 e4.

21. Gao, F., et al., The glutathione peroxidase gene family in Thellungiella salsuginea: genome-wide identification, classification, and gene and protein expression analysis under stress conditions. Int $J$ Mol Sci, 2014. 15(2): p. 3319-35.

22. Ursini, F., et al., Diversity of glutathione peroxidases. Methods Enzymol, 1995. 252: p. 38-53.

23. Mohammedi, K., et al., Glutathione peroxidase-1 gene (GPX1) variants, oxidative stress and risk of kidney complications in people with type 1 diabetes. Metabolism, 2016. 65(2): p. 12-9.

24. Jidong, Y., et al., GPx 1 knockdown suppresses chondrogenic differentiation of ATDC5 cells through induction of reductive stress. ACTA BIOCHIMICA ET BIOPHYSICA SINICA, 2017. 49(2): p. 110-118.

25. Foger-Samwald, U., et al., Molecular mechanisms of osteoporotic hip fractures in elderly women. Exp Gerontol, 2016. 73: p. 49-58.

26. Du, X.H., et al., SNP and mRNA expression for glutathione peroxidase 4 in Kashin-Beck disease. Br J Nutr, 2012. 107(2): p. 164-9.

27. A, P., Epigenetics as a unifying principle in the aetiology of complex traits and diseases. Nature, 2010. 465(7299): p. 721-7.

28. Skinner, M.K., Environmental epigenomics and disease susceptibility. EMBO Rep, 2011. 12(7): p. 6202.

29. van der Zwan, Y.G., et al., Seminoma and embryonal carcinoma footprints identified by analysis of integrated genome-wide epigenetic and expression profiles of germ cell cancer cell lines. PLoS One, 2014. 9(6): p. e98330. 
30. Ke, X., et al., Integrated analysis of genome-wide genetic and epigenetic association data for identification of disease mechanisms. Epigenetics, 2013. 8(11): p. 1236-44.

\section{Tables}

Table 1

Baseline characteristics of study population

\begin{tabular}{|lllll|}
\hline Characteristics & KBD $(\mathbf{n}=\mathbf{2 2 6})$ & Controls $(\mathbf{n = 2 4 8 )}$ & $\mathbf{t} / \mathbf{X}^{2}$ & $\mathbf{P}$ \\
\hline Age, $\overline{\mathrm{X}} \pm$ SD & $54.20 \pm 5.40$ & $53.50 \pm 6.20$ & 1.305 & 0.193 \\
\hline Gender, F/M & $116 / 110$ & $143 / 105$ & 1.914 & 0.167 \\
\hline
\end{tabular}

Table 2

The information of primers for mRNA expression detection

\begin{tabular}{|c|c|c|}
\hline Genes & Primer Sequence (5'-3') & Length(bp) \\
\hline \multirow[t]{2}{*}{ GPX1 } & Fw: ССТTССАСТTСАТСТGTССТG & \multirow[t]{2}{*}{149} \\
\hline & Rv: GATTCTTAGCCTCATCCGCC & \\
\hline \multirow[t]{2}{*}{ GPX3 } & Fw: TTCACGACATCCGCTGGAA & \multirow[t]{2}{*}{101} \\
\hline & Rv: CATCTTGACGTTGCTGACCGT & \\
\hline \multirow[t]{2}{*}{ GPX4 } & Fw: CTCTATGCAGGTTCCACTGTC & \multirow[t]{2}{*}{120} \\
\hline & Rv: TTCCСCAАACTTCTCCACAG & \\
\hline \multirow[t]{2}{*}{$\beta$-actin } & Fw: GAACGGTGAAGGTGACAGCAG & \multirow[t]{2}{*}{200} \\
\hline & Rv: GTGGACTTGGGAGAGGACTGG & \\
\hline
\end{tabular}


Table 3

Baseline characteristics of study population for $\mathrm{CpG}$ methylation

\begin{tabular}{|lllll|}
\hline Characteristics & KBD $(n=16)$ & Controls $(\mathbf{n = 1 6 )}$ & $\mathrm{t} / \chi^{2}$ & $\mathrm{P}$ \\
\hline Age, $\overline{\mathrm{x}} \pm$ SD & $54.26 \pm 5.61$ & $52.35 \pm 8.79$ & 0.733 & 0.469 \\
\hline Gender, F/M & $6 / 10$ & $8 / 8$ & 0.508 & 0.476 \\
\hline Degree, $\mathrm{n}(\%)$ & & & & \\
I & $1(6.25)$ & - & \\
II & $7(43.75)$ & - & \\
III & $8(50.00)$ & - & \\
\hline
\end{tabular}

Table 4

Baseline characteristics of study population for MSP-PCR

\begin{tabular}{|lllll|}
\hline Characteristics & KBD $(\mathbf{n = 8 5})$ & Controls $(\mathbf{n = 8 5})$ & $\mathbf{t} / \mathrm{\chi}^{2}$ & $\mathbf{P}$ \\
\hline Age, $\overline{\mathrm{x}} \pm \mathrm{SD}$ & $55.28 \pm 6.34$ & $54.32 \pm 8.74$ & 0.820 & 0.414 \\
\hline Gender, F/M & $25 / 60$ & $30 / 55$ & 0.672 & 0.412 \\
\hline
\end{tabular}

Table 5

Methylation rate of GPX3 between KBD patients and controls, $n$ (\%)

\begin{tabular}{|llllll|}
\hline Group & Methylation & Unmethylation & $\chi^{2}$ & P & OR $(95 \% \mathrm{Cl})$ \\
\hline KBD & $8(9.41)$ & $77(90.59)$ & 4.396 & 0.036 & $8.000(1.023,62.580)$ \\
Control & $1(1.18)$ & $84(98.82)$ & & & \\
\hline
\end{tabular}

\section{Figures}



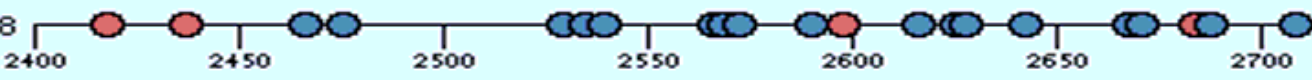

Product: 456 bp (from 2335 to 2790 ) with Coverage: 20

B chr5:151,020,246-151,020,701 456 bp. chr5:151,020,246-151,020,701 go

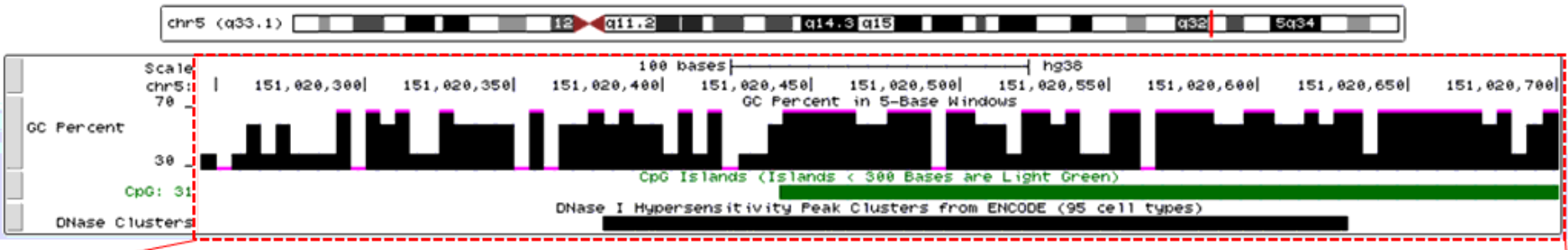

$\widehat{\text { G }}^{2335}$ GAATAAGAAATGCTTCCCAGAATGGAGACTTCCATCAGTTCTAGGGAGCTATTAGCCCCCTTGCCCTGGCTGTAATGGAGACCG ${ }^{[1]}$ C TGTGTCTGCCTCCTTTCG ${ }^{[2]}$ CACTTTGGAGCCAAAAGAGGAAGGGACC ${ }^{[3]}$ CCTCCCACG $^{[4]}$ TCCACAGGGACCTGACTTCCACCTCTCTG

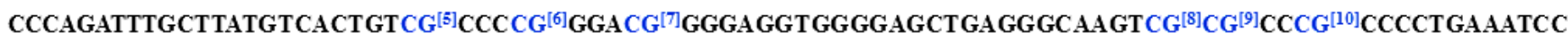
CAGCCG $^{[11]}$ CCTAGCG $^{[12]}$ ATTGGCTGCAAGGGTCTCG ${ }^{[13]}{ }_{\text {GCTTGGCCG }}{ }^{[14]}$ CG $^{[15]}{ }_{\text {GATTGGTCACACCCG }}{ }^{[16]}$ AGGGCTTGAAAGGTGGCTGG GAGCG $^{[17]}$ CCG $^{[18]}$ GACACCTCAGACG $^{[19]}$ GACG $^{[20]}$ GTGGCCAGGGATCAGGCAGCG ${ }^{[21]}$ GCTCAGGCG ${ }^{[22]}$ ACCCTGAGTGTGCCCCCACCCC $\mathrm{G}^{[23]}$ CCATGGCCCG $^{[24]}$ GCTGCTGCAGGCG $^{[25]}$ TCCTGCCTGCTTTCCCTGCTCCTG $^{2790}$

Figure 1

The information of $\mathrm{CpG}$ methylation sites in primer GPX3 \#8 and the sequence of target fragment in the promoter region of GPX3 (A: The dots represent the $\mathrm{CpG}$ sites to be tested in the target fragment; $\mathrm{B}$ : The blue bases represent $\mathrm{CpG}$ sites to be tested). 
$0 \% 000000000100 \%$ Not analyzed

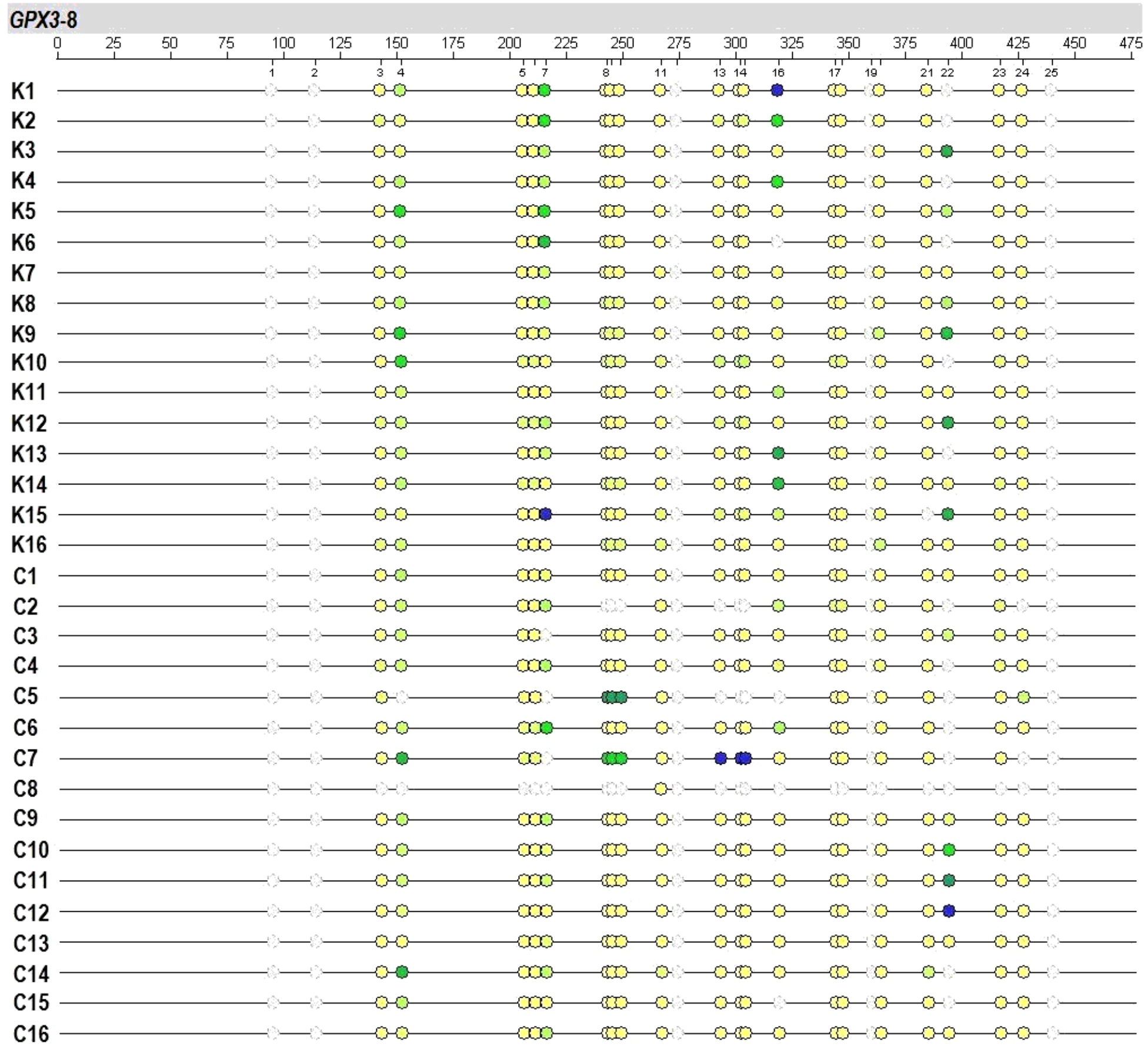

\section{Figure 2}

Methylation levels of $\mathrm{CpGs}$ in the promoter region of GPX3 in KBD and Controls (The color of the dots indicates the methylation level. The darker the color, the higher the methylation level; otherwise, the lower the methylation level. The numbers 1-25 in the figure represent CpGs GPX3-8_CpG_1-25; KBD: KBD patients, C: healthy control group). 


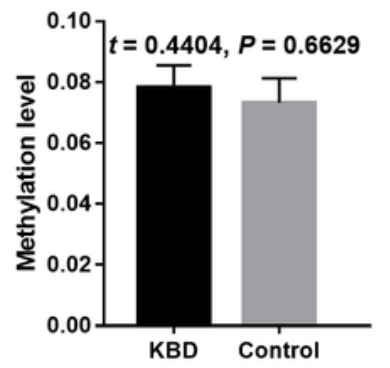

(a) GPX3 - 8_CpG_3

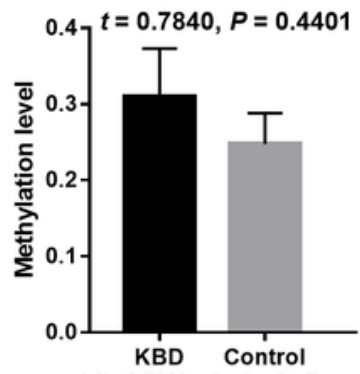

(d) GPX3 - 8_CPG_7

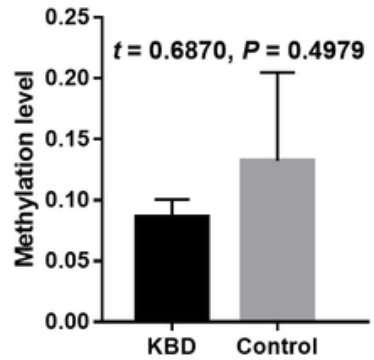

(g) GPX3 - 8_CpG_13.14.15

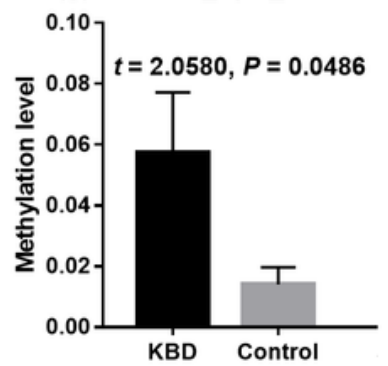

(j) GPX3 - 8_CpG_20

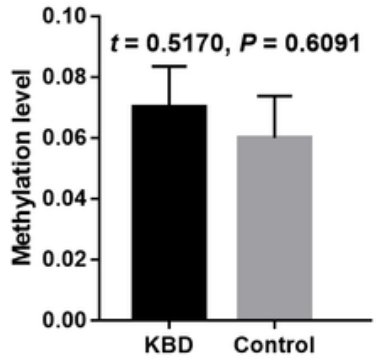

(m) GPX3 - 8 CpG 23

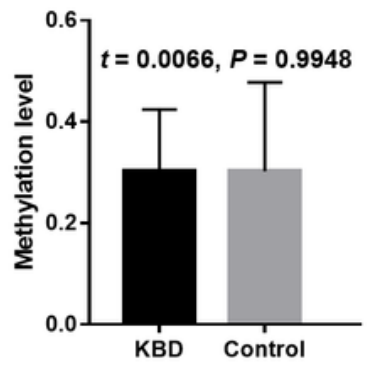

(b) GPX3 - 8_CPG_4

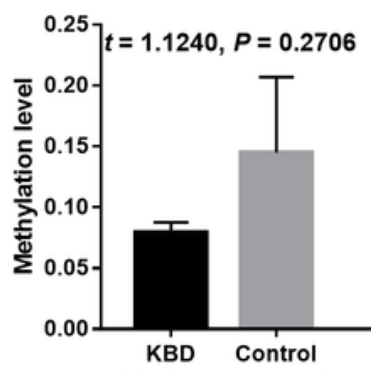

(e) GPX3 - 8_CpG_8.9.10

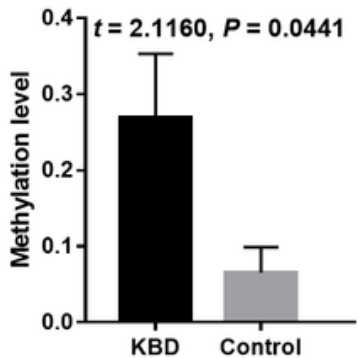

(h) GPX3 - 8_CpG_16

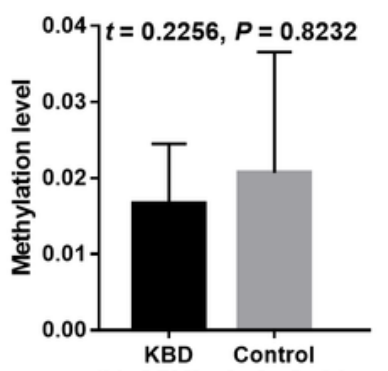

(k) GPX3 - 8_CPG_21

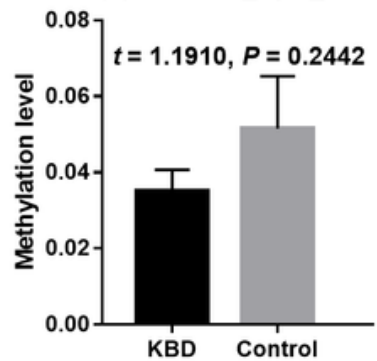

(n) GPX3 - 8 CpG 24

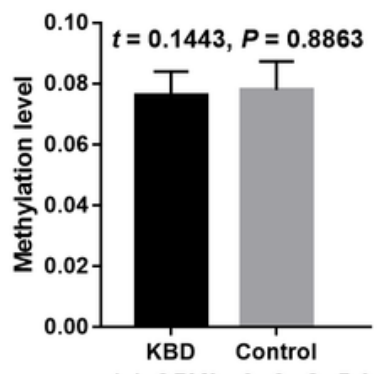

(c) GPX3 - 8_CpG_5.6

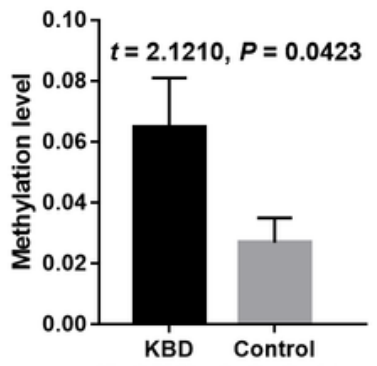

(f) GPX3 - 8_CpG_11

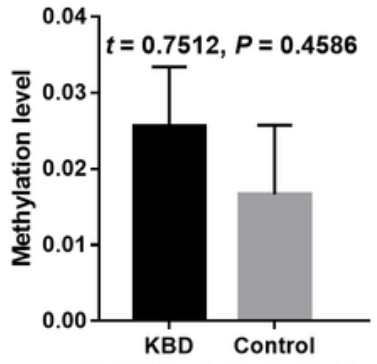

(i) GPX3 - 8_CpG_17.18

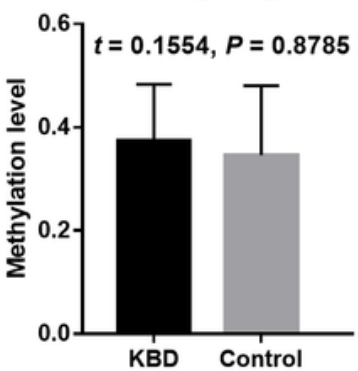

(I) GPX3 - 8_CPG_22

\section{Figure 3}

Comparison of methylation levels of four CpGs in the promoter region of GPX3 between KBD and Controls. 


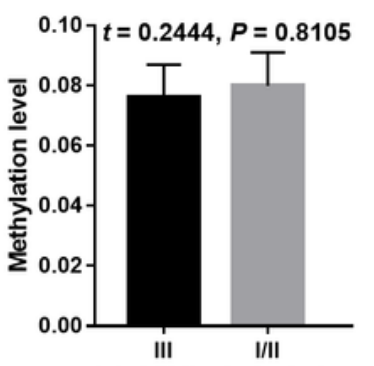

(a) GPX3 - 8_CpG_3

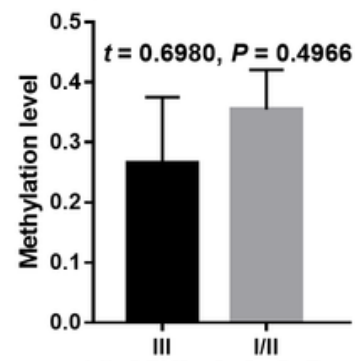

(d) GPX3 - 8_CpG_7

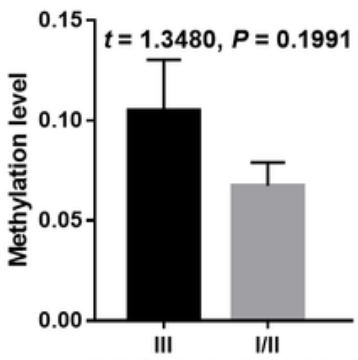

(g) GPX3 - 8_CpG_13.14.15

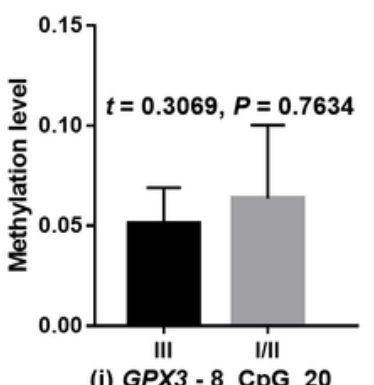

(j) GPX3 - 8_CpG_20

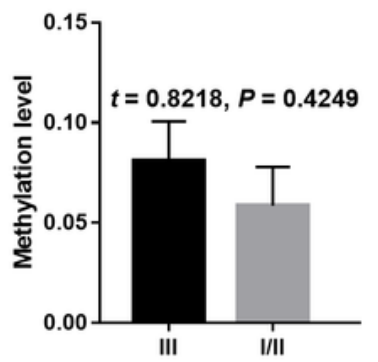

(m) GPX3 - 8_CpG_23

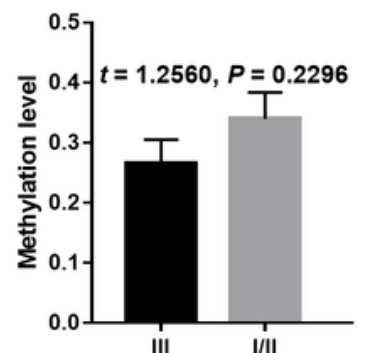

(b) GPX3 - 8_CpG_4

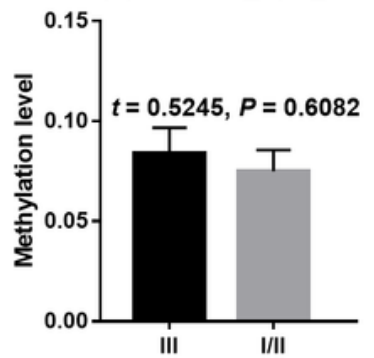

(e) GPX3 - 8_CpG_8.9.10

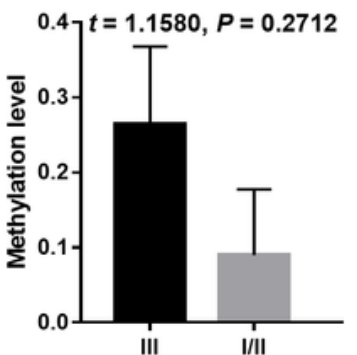

(h) GPX3 - 8_CpG_16

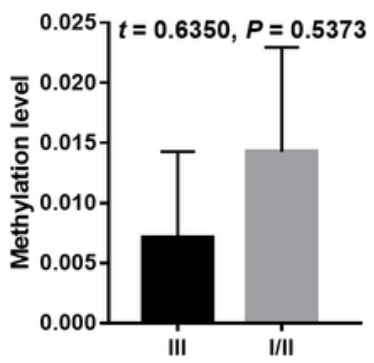

(k) GPX3 - 8_CPG_21

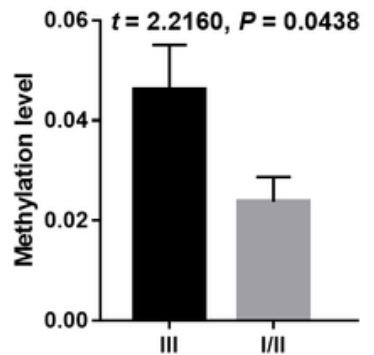

(n) GPX3 - 8_CpG_24

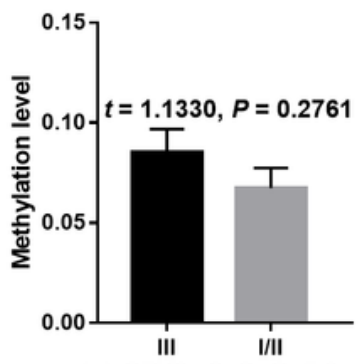

(c) GPX3 - 8_CpG_5.6

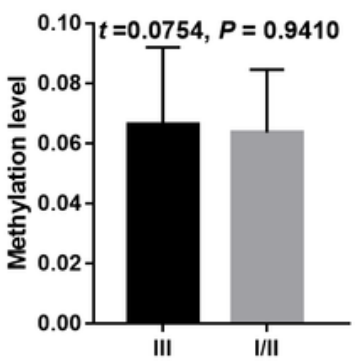

(f) GPX3 - 8_CpG_11

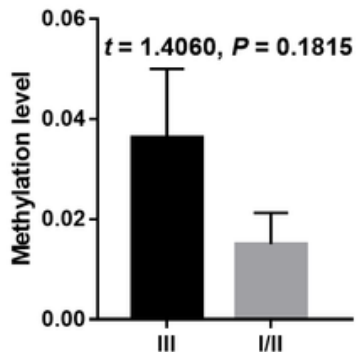

(i) GPX3 - 8_CpG_17.18

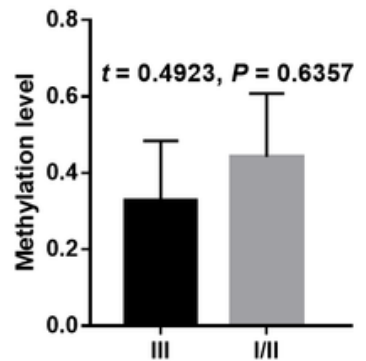

(I) GPX3 - 8_CPG_22

Figure 4

Comparison of methylation levels of $\mathrm{CpGs}$ in the promoter region of GPX3 between KBD patients with different degree. 


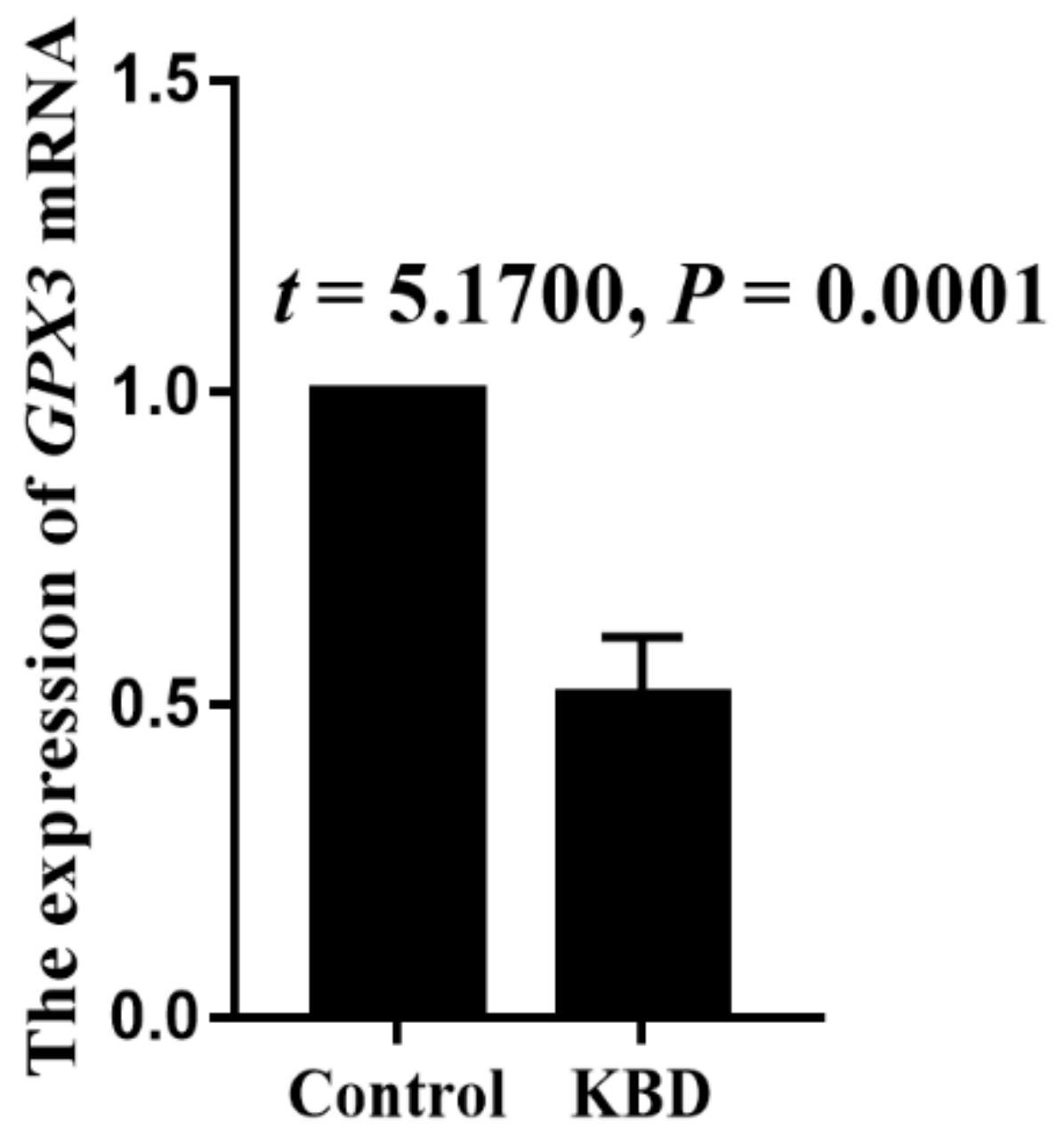

Figure 5

GPX3 mRNA levels in peripheral blood of KBD patients.

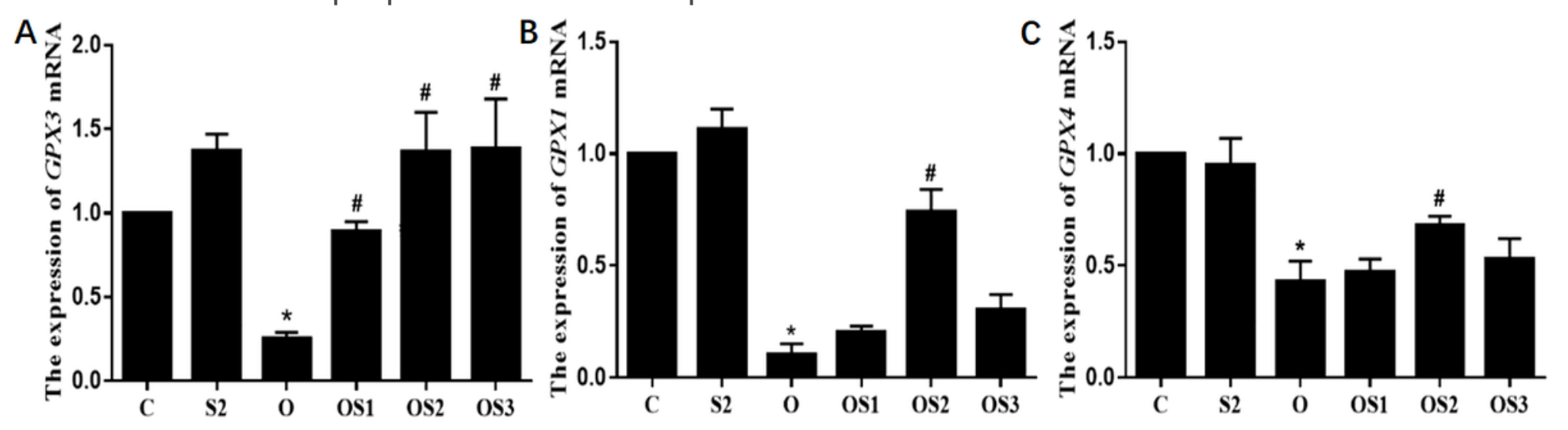

Figure 6

GPX1, GPX3, GPX4 mRNA levels in in chondrocytes treated by tBHP and Na2SeO3 (C: control group; S2: treated by $0.10 \mu \mathrm{g} / \mathrm{mL}$ Na2Se03; O: treated by $150 \mu \mathrm{mol} / \mathrm{L}$ tBHP; OS1: treated by $0.05 \mu \mathrm{g} / \mathrm{mL}$

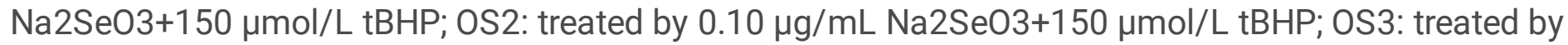

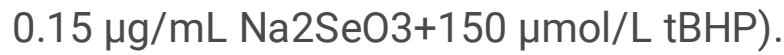



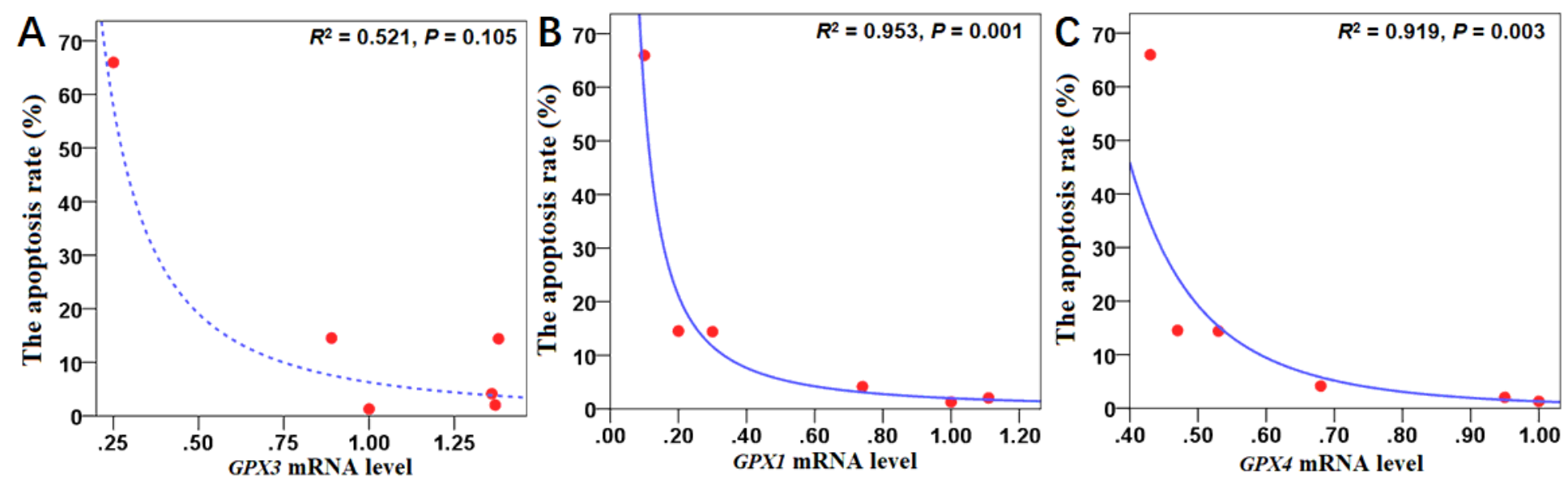

Figure 7

The correlation between GPX1, GPX3, GPX4 mRNA expressions and apoptosis rate of chondrocytes (C: control group; S2: treated by $0.10 \mu \mathrm{g} / \mathrm{mL}$ Na2Se03; O: treated by $150 \mu \mathrm{mol} / \mathrm{L}$ tBHP; OS1: treated by 0.05

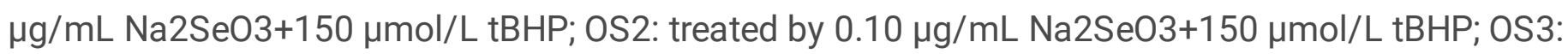

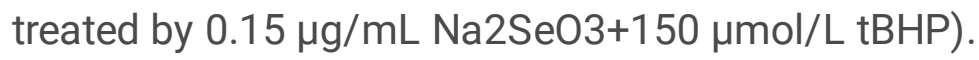




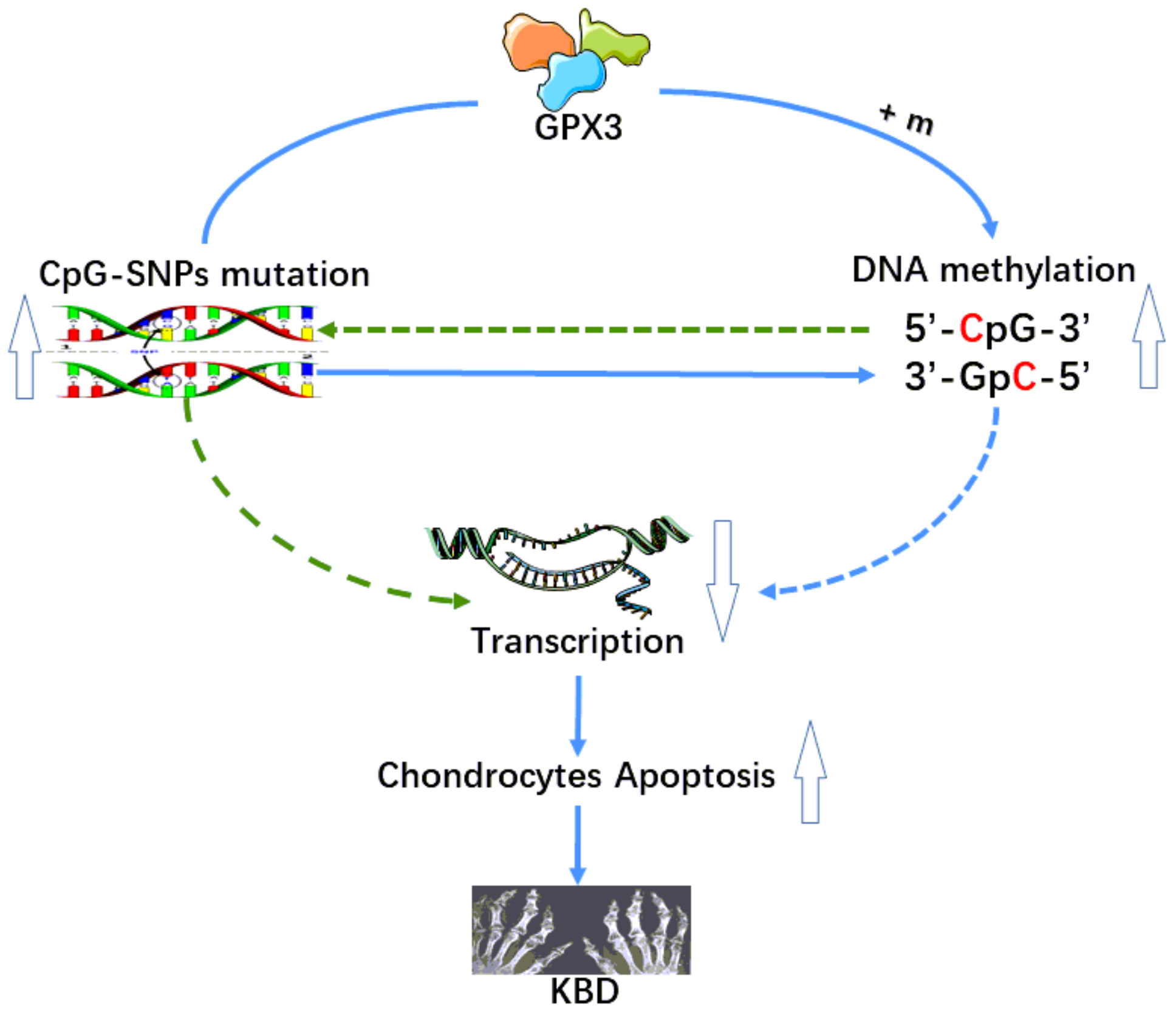

Figure 8

New mechanism in the development of KBD. 